\title{
ARTIGO
}

doltps://doi.org/10.22481/praxisedu.v16i39.6295

\section{IDENTIDAD Y ESPIRITUALIDAD MAYA EN LA ESCUELA DE AGRICULTURA ECOLÓGICA $U$ YITS KA 'AN EN MANÍ, YUCATÁN, MÉXICO}

\author{
MAYAN IDENTITY AND SPIRITUALITY IN THE $U$ YITS KA'AN ECOLOGICAL \\ AGRICULTURE SCHOOL IN MANÍ, YUCATÁN, MÉXICO
}

IDENTIDADE E ESPIRITUALIDADE MAIAS NA ESCOLA DE AGRICULTURA ECOLÓGICA $U$ YITS KA'AN EM MANÍ, YUCATÁN, MÉXICO

\section{Rosa López Valentín}

El Colegio de la Frontera Sur - México Universidad Estatal de Ceará - Brasil

Peter Michael Rosset

El Colegio de la Frontera Sur - México

Carla Beatriz Zamora Lomelí

El Colegio de la Frontera Sur - México

Omar Felipe Giraldo Palacio

El Colegio de la Frontera Sur - México

María Virginia González Santiago

Universidad Autónoma de Chapingo - México

Resumen: Este articulo tiene como objetivo, analizar la importancia de la espiritualidad y la identidad colectiva, en la propuesta de la (re)construcción comunitaria de la Escuela de Agricultura Ecológica $U$ Yits Ka'an, localizada en el municipio de Maní, en el estado de Yucatán, México. Para esta investigación, se utilizó una metodología de corte cualitativo a través de trabajo etnográfico. Los resultados muestran que la estrategia que sigue la escuela U Yits Ka'an, para construir un ambiente donde se pueda vivir el Máalob Kuxtal (buen vivir maya), se basa en la inclusión de dimensiones espirituales y de identidad maya y campesina, para priorizar lo colectivo en la solución de problemas y re valorar el ser, antes del hacer en su cotidianidad. Estos procesos tienen como consecuencia la construcción humana y etnocentrada basada en una vida buena plena y digna o Máalob Kuxtal, que es un modo de vida, dentro de las comunidades mayas.

Palabras clave: Identidad colectiva; espiritualidad maya, Recuperación histórica, Escuela campesina, Comunitariedad

Abstract: The purpose of this article is to analyze the importance of spirituality and collective identity for the (re)construction of community at the U Yits Ka'an Ecological Agriculture school located in the 
municipality of Maní, in the southern part of the state of Yucatán, Mexico. A qualitative methodology was used based on ethnographic work. The results show that the strategy followed by the U Yits Ka'an school, to build an environment where the Kuxtal Ma'alob (mayan buen vivir) can be lived, is based on the inclusion of spiritual dimensions and Mayan and peasant identity, in order to prioritize the collective in the solution of problems and to re-value the being, before the doing in their daily lives. These processes have as a consequence the human and ethnocentric construction based on a good, full and dignified life or Ma'alob Kuxtal, which is a way of life within Mayan communities.

Key words: Collective identity; Mayan spirituality, Historical recovery, Peasant school, Comunitariety

Resumo: O objetivo deste artigo é analisar a importância da espiritualidade e da identidade coletiva na proposta de (re)construção comunitária da Escola de Agricultura Ecológica U Yits Ka'an, localizada no município de Maní, no estado de Yucatán, México. Para esta pesquisa, foi utilizada uma metodologia de corte qualitativo através de trabalho etnográfico. Os resultados mostram que a estratégia seguida pela escola U Yits Ka'an, de construir um ambiente onde o Kuxtal Ma'alob ( bem viver maya) possa ser vivido, baseia-se na integração das dimensões espirituais e da identidade maia e camponesa, a fim de priorizar o coletivo na solução dos problemas e revalorizar o ser, antes de fazê-lo em suas vidas diárias. Estes processos têm como consequência a construção humana e etnocêntrica baseada numa vida boa, plena e digna ou Ma'alob Kuxtal, que é um modo de viver dentro das comunidades maias.

Palavras-chave: Identidade coletiva; Espiritualidade Maya; Recuperação histórica; Escola camponesa; Comunidade

\section{Introducción}

El mundo atraviesa por una crisis que se manifiesta en aspectos económicos ecológicos, espirituales, alimentarios, entre otros, las cuales no pueden ser entendidas individualmente, sino como consecuencia del modelo civilizatorio (ESTERMANN, 2012). En México, son las áreas rurales las que sufren especial presión ante los efectos de la crisis, que, aunado a otros factores como el incremento de la violencia y el narcotráfico, los cambios en el uso de suelo y la migración, han ocasionado que las familias campesinas a menudo enfrenten el deterioro de sus entornos socioculturales y ambientales. En respuesta a estos desafíos, hay diversos procesos locales y regionales que intentan lograr la recuperación del tejido comunitario para alcanzar colectivamente una alternativa al paradigma occidental de desarrollo. Un ejemplo de ello es la Escuela de Agricultura Ecológica U Yits Ka'an (palabras mayas que quieren decir "rocío que cae del cielo"), un centro de formación agroecológica para campesinos y campesinas mayas en el estado de Yucatán, creado en 1991. 
Un grupo de sacerdotes católicos Inspirados en la teología de la liberación ${ }^{1}$, junto con campesinos mayas, campesinos guatemaltecos practicantes del método campesino a campesino $(\mathrm{CaC})$ y algunos profesores de las universidades autónomas de Chapingo (UACh) y Yucatán (UADY), dieron inicio al proceso de trabajo de la escuela $U$ Yits $K a$ 'an.

Desde entonces este espacio ha tomado como referentes para su sistema de formación dos elementos: la revalorización de la cultura tradicional y la agroecología o agricultura ecológica (MACOSSAY; AGUILAR; CASTILLO; CEBALLOS; ZAPATA, 2005). Del mismo modo, las bases de la teología de la liberación, en combinación con las de una teología indígena, continúan vigentes dentro de sus procesos educativos, mismos que abordan la espiritualidad a partir de la recuperación de los rituales mayas y la resignificación de la identidad.

Ante escenarios como los inicialmente descritos, es importante que las propuestas que emergen desde escuelas como la de Agricultura Ecológica U Yits Ka'an no solo deban ser reconocidas, sino también sistematizadas y visibilizadas, para que sus experiencias aporten y dialoguen con las de sus similares en otros contextos territoriales. En ese sentido, el objetivo es analizar los alcances, estrategias y mecanismos pedagógicos, metodológicos y espirituales implementados por la escuela para aportar a la construcción de la comunidad como horizonte de vida.

Para ello, se desarrolló una estrategia metodológica de corte cualitativo, a través de técnicas de investigación como la observación participante y entrevistas a profundidad y semiestructuradas. El análisis de la investigación se realizó a partir de dos categorías generales: la espiritualidad y la identidad colectiva.

Inicialmente presentamos un breve recorrido histórico de la fundación y el contexto de la escuela para entender su dinámica. Posteriormente exponemos los conceptos clave para abordar el tema de estudio. Continuamos con la exposición de los resultados de la investigación; y, finalmente, abrimos la discusión acerca de la importancia de la experiencia concreta de la escuela, para apoyar o enriquecer otro tipo de procesos que buscan dar respuestas locales ante la crisis civilizatoria.

Proponemos que la espiritualidad y la identidad colectiva son base para la (re)construcción comunitaria y la puesta en marcha de procesos agroecológicos. Como premisa para la creación de un contexto en el que se pueda desarrollar una vida buena plena y digna o Máalob Kuxtal, en el área de influencia de la escuela de Agricultura Ecológica U Yits Ka'an.

\footnotetext{
${ }^{1}$ El proceso reflexivo que surgió en América Latina durante la década de los sesenta y que pretende otorgarle a la iglesia católica un rostro más humano que pretende transformar la realidad de los menos favorecidos (BERRYMAN, 2003).
} 


\section{Contexto histórico de la investigación}

Al sur del estado de Yucatán se encuentra el municipio de Maní, lugar en que se estableció la escuela de agricultura ecológica U Yits Ka'an en la década de los noventa.

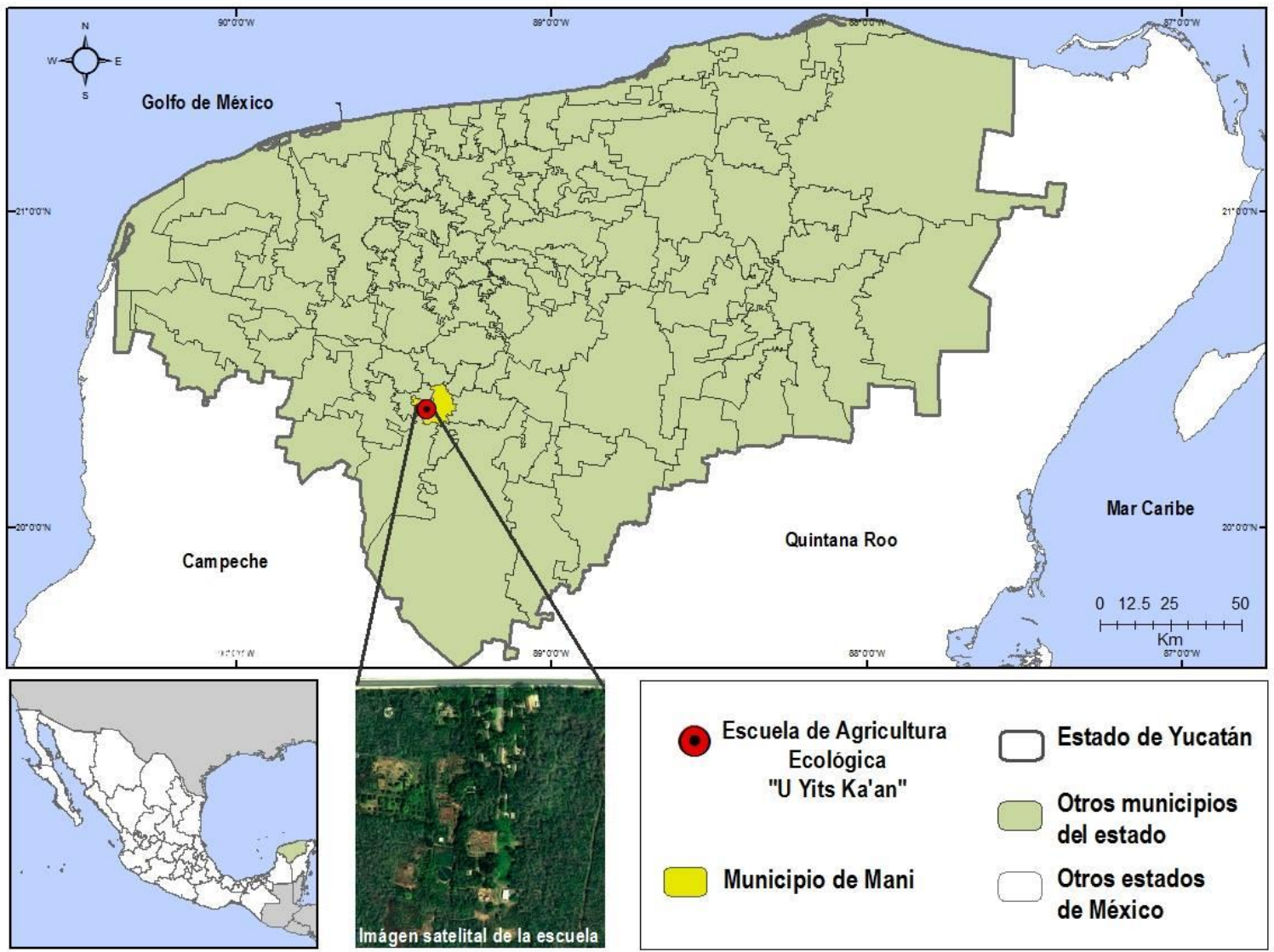

Figura 1. Ubicación de la escuela. Fuente: elaboración propia.

Para entender la complejidad de su planteamiento, la ubicaremos tanto geográfica como históricamente. Entender la historia de los mayas peninsulares, en particular de la evangelización en la época colonial en Maní, nos permitirá visibilizar el proceso de recuperación de la identidad maya como acto de resistencia.

El municipio de Maní es un lugar emblemático de la resistencia maya, ya que aquí se llevó a cabo el "auto de fe de Maní", una de las escenas más cruentas de la conquista en el continente. Por esta razón la existencia de la escuela en este lugar es ya un acto de rebeldía, persistencia y resistencia. Así como también entender la dimensión espiritual e ideológica de la escuela a partir de su génesis. 
El origen del pueblo maya que se asentó en el estado de Yucatán, se remonta a 8,000 años aproximadamente. En esta región tres linajes determinaban los aspectos sociales, económicos, políticos y militares: los Cocomes en Mayapán, los Itzaes en Chichen Itzá, y los Xiues en Uxmal (Kú-May, 2005).

La ciudad de Maní fue fundada entre 1224 y 1244 y fue gobernada por el linaje Xiu. En vísperas de la conquista española, Maní era el Kuchkabal (provincia), más importante de la península de Yucatán por su posición política, su territorio y su gran densidad demográfica (SUÁREZ, 2014). En 1547 los frailes llegaron a Maní para evangelizar a sus habitantes, construyeron escuelas para enseñar a leer, escribir y cantar a los niños indígenas, además se les instruía para que asistieran a los frailes en las celebraciones religiosas. Los conquistadores exhortaron a los caciques a escuchar su doctrina y obligaron a los pueblos mayas a construir templos donde hiciera falta; recalcándoles que la religión cristiana era la única puerta para entrar al cielo y que quien siguiera practicando sus cultos pagaría la ofensa en el infierno (CASTRO GUZMÁN; CANO; CENTURIÓN, 2017).

En Maní se construyó el convento de San Miguel Arcángel, lugar que el 12 de julio de 1562 fue el escenario del “Auto de fe de Maní”; cuando a Fray Diego de Landa le notificaron el hallazgo de una cueva con animales sacrificados, además de otros elementos que indicaban una práctica clandestina de adoración, acciones que fueron consideradas como un sacrilegio. El castigo fue apresar indígenas, en su mayoría pertenecientes a la élite, torturándolos públicamente; se quemaron códices, destruyeron esculturas de dioses mayas y desenterraron los cuerpos de aquellos que en vida habían cometido idolatría a ojos de los frailes (Suárez, 2014).

Esta noche se convierten en cenizas ocho siglos de literatura maya. En estos largos pliegos de papel de corteza, hablaban los signos y las imágenes: contaban los trabajos y los días, los sueños y las guerras de un pueblo nacido antes que Cristo. Con pinceles de cerdas de jabalí, los sabedores de cosas habían pintado estos libros alumbrados, alumbradores, para que los nietos de los nietos no fueran ciegos y supieran verse y ver la historia de los suyos, para que conocieran el movimiento de las estrellas, la frecuencia de los eclipses y las profecías de los dioses, y para que pudieran llamar a las lluvias y a las buenas cosechas de maíz. (GALEANO, 1991, p. 118).

Lo escrito por Galeano retrata la importancia de la recuperación de la memoria, la necesidad de recuperar la identidad maya y que el pueblo se reencuentre con la grandeza de su cultura. 
Surgimiento de la escuela U Yits Ka'an

En la década de los setenta, organizaciones no gubernamentales (ONG) internacionales comenzaron a desarrollar la llamada "agricultura sustentable" en Mesoamérica. Estas prácticas fueron apropiadas por un grupo de campesinos mayas kaqchikeles de la comunidad de San Martín Jilotepeque, departamento de Chimaltenango, en Guatemala, quienes comenzaron a practicar estas técnicas, y prácticas ancestrales recuperadas, en sus parcelas y después enseñaron a otros campesinos locales. Así se originó el Movimiento de Campesino a Campesino, que se expandió después por Mesoamérica (HOLT-GÍMENEZ, 2008). Este movimiento es un antecedente importante en el surgimiento de la escuela $U$ Yits $\mathrm{Ka}$ 'an, ya que en la década de los ochenta un grupo de campesinos mayas originarios de San Martín Jilotepeque llegaron como refugiados al estado de Yucatán, huyendo de la guerra en Guatemala de la que fueron víctimas. Estos campesinos comenzaron a impartir talleres de campesino a campesino sobre temas relacionados con la conservación de suelos, como la labranza de conservación y la elaboración de compostas, en comunidades del estado. En este proceso conocieron a un grupo de sacerdotes inmersos en la teología de la liberación, quienes empezaron a difundir la labor de estos campesinos en sus parroquias, trabajando coordinadamente; esta relación contribuyó a la construcción del proyecto de la escuela.

Así, en el año de 1991, un grupo de sacerdotes adscritos a la teología de la liberación y la pastoral de la tierra, que trabajaban en varias localidades del estado de Yucatán, propusieron la creación de un centro de formación para campesinos y campesinas con la finalidad de promover procesos sustentables de alternativas al desarrollo, tomando en cuenta dos elementos: la revalorización de la cultura tradicional y la agricultura ecológica (MACOSSAY et al., 2005). En 1994 se compra un terreno a las afueras de la cabecera municipal de Maní, gracias al financiamiento de la fundación alemana Misereor, institución para la cooperación internacional al desarrollo que pertenece a la iglesia católica. Con los recursos disponibles se construyó la infraestructura necesaria para operar una escuela. Las instalaciones se terminaron a finales de 1995. Ese mismo año, en un esfuerzo conjunto entre los sacerdotes y profesores de algunas universidades y ONG, se elaboraron los primeros planes de estudio de la escuela (MACOSSAY et al., 2005). En la escuela se impartieron clases de 1996 al 2005 a campesinos/as de la región, en un sistema de seminternado. En el 2005 concluye el financiamiento de la fundación Misereor haciendo inoperable el sistema de internado. En el año 2009 se creó un sistema de subsedes, con el objetivo de tener mayor contacto con las comunidades de la región, y tener un vínculo 
más estrecho con las familias campesinas, mediante el establecimiento de centros de formación coordinados por los mismos egresados (HERNÁNDEZ, 2015; MELECIO, 2015). Muchos han sido los actores internos y externos y aliados que ha tenido la escuela, entre ellos los sacerdotes católicos, los campesinos mayas yucatecos y guatemaltecos, instituciones educativas, ONG nacionales e internacionales, así como fundaciones que, sin duda, han abonado desde su campo de acción para hacer posible este proyecto, que se ha modificado y adaptado con el paso del tiempo (ver Figura 2). La cooperación internacional ha sido uno de los aliados más importantes, en particular las fundaciones Misereor y Adveniat (agencias que apoyan el trabajo pastoral de la Iglesia Católica en América Latina), las cuales han jugado un rol primordial en el sostenimiento de la escuela y reflejan el carácter pastoral con que se ha configurado.

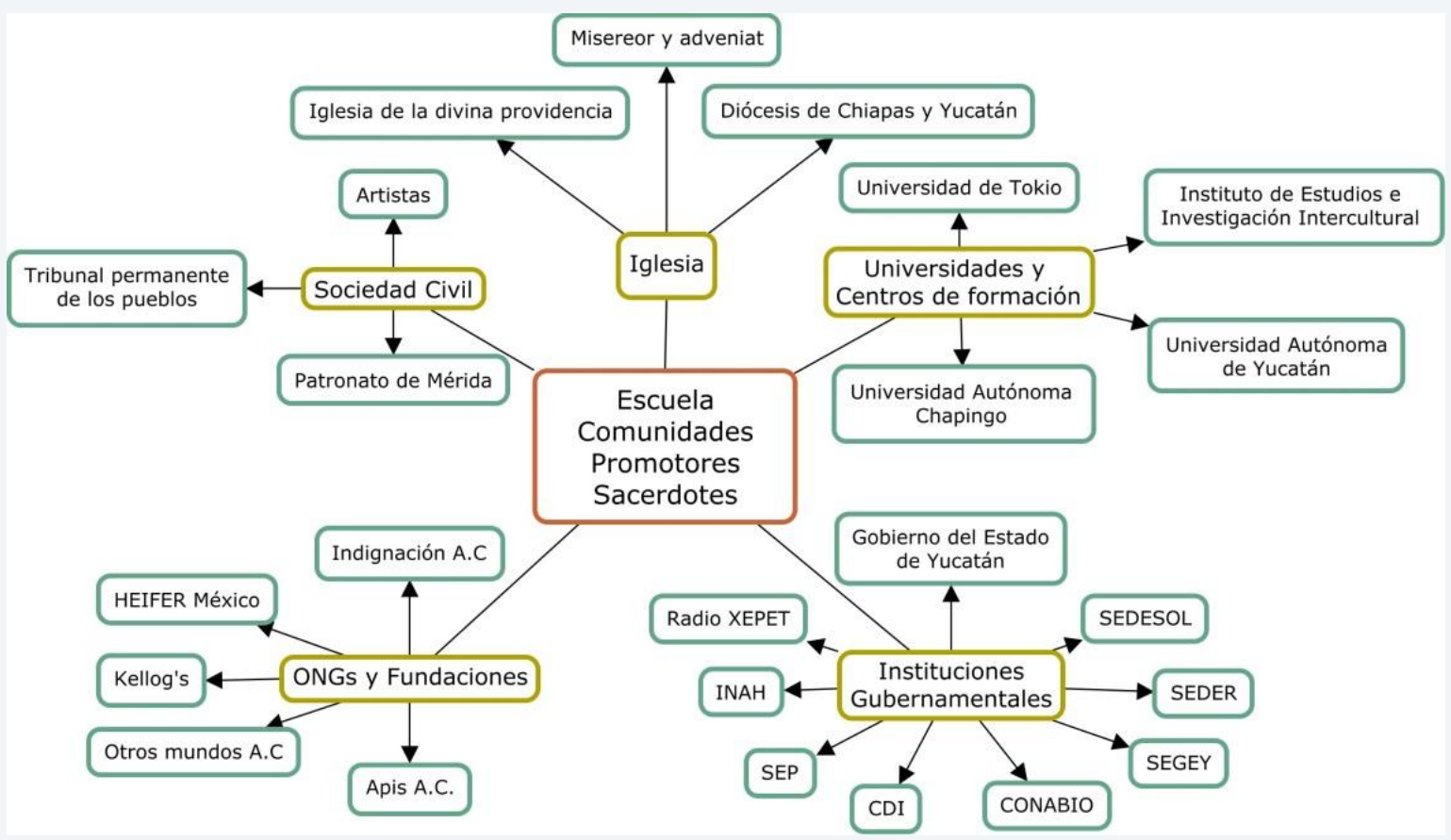

Figura 2. Actores y aliados que han colaborado con la escuela. Fuente: elaboración propia.

La base teológica y educativa de la escuela

Las escuelas campesinas de agroecología (ECAS), se plantean como mecanismos de dialogo entre una ideología productiva y el conocimiento local. Surgen como una opción para la integración y aplicación de tecnologías a contextos específicos de la vida rural con valores campesinos, para promover alternativas endógenas al desarrollo y posibilitar la reapropiación territorial, así como la intervención en dimensiones sociales y políticas, particularmente en relación con la soberanía alimentaria. Hacen uso de la sabiduría local para reconstruir un 
modelo de producción sustentable localmente situado en una ideología indígena y campesino (ÁLVAREZ, 2010).

La escuela U Yits Ka'an se ubica como una escuela de capacitación agroecológica o escuela de agroecología. El proceso de formación está basado en el "aprendizaje significativo", que privilegia la práctica sobre la teoría y valora los saberes ancestrales de la cultura campesina maya, con base en una pedagogía de enseñanza de campesino a campesino (CEBALLOS, 2014). Las escuelas campesinas implican una forma alternativa de pensar, hacer y actuar en relación con la educación formal (MUÑOZ, 2013). Los procesos educativos y formativos relacionados con productores, campesinos e indígenas buscan recuperar y reafirmar la importancia de los saberes locales como un aporte de intervención propia frente a una propuesta que originalmente es disciplinaria (técnico/productiva), pero posteriormente desarrolla una reflexión colectiva y genera las condiciones de un intercambio de saberes.

Martínez-Torres y Rosset, (2016) definen el diálogo de saberes como:

La construcción colectiva de significación emergente, basada en el diálogo establecido entre personas o pueblos cuyas experiencias, cosmovisiones y cuando enfrentan los nuevos desafíos comunes de un mundo cambiante. Dicho diálogo se apoya en el intercambio entre las diferencias y en la reflexión colectiva. A menudo, ello propicia la recontextualización y la re-significación, lo cual da lugar a saberes y significados emergentes, que se relacionan con historias, tradiciones, territorialidades, experiencias, procesos y acciones de los distintos pueblos. Las nuevas y colectivas comprensiones, significados y saberes, pueden llegar a constituir la base para acciones de resistencia colectivas, y para la construcción de procesos colectivos nuevos. (p.26).

Este diálogo de saberes, expresado en el caso específico de U Yits Ka'an, tiene un carácter particular, no solamente de intercambio con sectores académicos y gubernamentales sino entre campesinos/as, que alimenta sus propuestas pedagógicas con un sentido diferente de la práctica y un marco teórico decolonial y de frontera, lo que extiende sus alcances más allá de ser estrictamente pedagógica a un modelo sociopolíticamente situado de interpretación de la realidad.

Ejemplo de ello es el dialogo construido con la Red Nacional de Escuelas Campesinas, del cual forman parte la escuela desde el año 2003. Esta red opera a través de enlaces regionales y la escuela ha sido uno de estos. Desde la participación de la escuela en estos espacios se ha contribuido a la discusión en torno a la definición del concepto de escuela campesina y cuáles deben ser los principios rectores de estas. En estos encuentros se ha insistido en la importancia de valorar la cultura y reafirmar la identidad campesina e indígena. Se acordaron también los 
principios que tienen que dar sustento a las escuelas de campo, tales como la espiritualidad, la equidad, el comunitarismo, la comunidad, la interculturalidad, la ética, la sustentabilidad y la solidaridad, a la vez que se contrajo el compromiso de defender y conservar la Madre tierra (MATA, 2013).

La escuela $U$ Yits $K a$ 'an aporta desde su práctica hacia otras propuestas, profundizando sus sentidos, ampliando desde sus horizontes productivistas y entrelazando sus actividades o ejercicios de interpretación de la realidad e incidencia en otros espacios, por ejemplo, en la construcción de la teología indígena máyense con las comunidades eclesiales de base en el estado de Chiapas, surgidas desde la teología de la liberación.

La propuesta de la teología de la liberación fue generada en el marco de la II Conferencia Episcopal de Latinoamérica, en Medellín, Colombia, en 1968; hacia la construcción ideológica de la Iglesia cambiando su estructura vertical y jerárquica por una iglesia que acompañe a los pobres y oprimidos del sur global (SEGUNDO, 1978). Surge así la propuesta de una iglesia en y desde los pobres, organizada en Comunidades Eclesiales de Base (CEBs), con fuerte acento en lo popular y laico y en una perspectiva política social cristiana, con conciencia social, de democratización de las bases y de ruptura de la neutralidad, en forma de movimiento popular y en confrontación a las dictaduras implantadas en todo Latinoamérica (BOFF, 1990).

La teología de la liberación fundamenta su práctica pastoral en la reconstrucción histórica cultural, la reflexión y el análisis político, ideológico y económico, en busca de caminos de liberación de los pueblos a partir de la concientización, la organización social y la reconstrucción comunitaria. En este proyecto, obispos, sacerdotes y laicos trabajan conjuntamente para promover una relación de sabiduría, religiosidad popular, construcción de fe y de esperanza (BOERO, 1984). A pesar de que la teología de la liberación volteó a ver a los pobres, para los pueblos indígenas es una propuesta que no alcanza a enmarcar la complejidad de sus realidades y cosmovisiones. De ahí la importancia y necesidad de construir una teología por y para los indígenas, considerando los elementos propios de su origen y su visión del mundo (ARIAS; CARRASCO, 1998).

La teología indígena surge entonces de la teología de la liberación que, en su proceso de liberación, propicia la construcción de sujetos conscientes de su capacidad transformadora, y los motiva como sujetos a desarrollar su propia visión de la vida, de la necesidad de justicia y coloca en la fortaleza de la construcción histórica su identidad, así como la construcción comunitaria como su fin último (CAMPOS, 2007). Hay una serie de elementos con los que se 
construye la teología indígena que es importante resaltar: por principio, la espiritualidad de la pobreza. Esta enorme fuente de fe y esperanza que surge de hombres y mujeres humildes, sensibles al dolor humano, al despojo y a la falta de oportunidades, pero también cercanos a la solidaridad, la recuperación de la dignidad, la búsqueda de justicia y la fraternidad. Por esto, la teología de la liberación, pero particularmente la teología indígena que camina junto con los más pobres y posee una fortaleza espiritual que refuerza la unidad comunitaria (ARIAS; CARRASCO, 1998).

Así mismo, la cosmovisión y espiritualidad indígena son fuentes de fe muy importante: su visión de armonía global, su sentido de respeto a todos los elementos, basado en el reconocimiento del pasado y enarbolando una visión de futuro que se afianza en su conciencia del presente, consolidando un sentido de pueblo que está vivo, que llega hasta el corazón de su identidad más profunda. La espiritualidad indígena está enlazada fuertemente con su raíz histórica; gira en torno a su experiencia de Dios Padre-Madre Tierra, que es proveedor, cuna y fin último; encuentra su fuerza en el vínculo con la tierra y el territorio que defienden con toda su espiritualidad y fuerza incansable para proteger en lo que creen (ARIAS; CARRASCO, 1998).

La teología de los pueblos indígenas es relacional, porque coincide con principios científicos, al asumir que la vida es un conjunto de relaciones internas y con su entorno. Lo que se aplica en términos prácticos en funciones como la alimentación y reproducción, entendido de esta forma la teología indígena como un motor de cambio y de transformación de este mundo (LÓPEZ, 2013).

Podemos afirmar, entonces, que la escuela tiene como prioridad la liberación de los campesinos y campesinas de la región maya a partir del trabajo de reconstrucción, resignificación histórica y teológica; y que a partir de esta base ideológica se incluyen y transforman los conocimientos técnicos, agroecológicos, forestales, artesanales y de medicina tradicional, entre muchas otras actividades, que aplican de manera particular en coherencia con su cotidianidad y estilo de vida. Fundar entonces una escuela campesina que a través de la teología indígena máyense y la reconstrucción histórica de su cultura recupere la identidad de una comunidad, no es casual. 


\section{La estrategia de la escuela de U Yits Ka'an para la (re)construcción comunitaria}

A través del estudio de su organización, articulación e interrelación de sus proyectos con miras a una resignificación identitaria, identificamos como su fin último un "buen vivir", al que aspira llegar mediante la reconstrucción de la comunidad ${ }^{2}$.

a) Estructura y función de la escuela.

Para su funcionamiento confluyen un conjunto de elementos importantes: por un lado, la relación estrecha que se dio al inicio entre campesinos mayas y guatemaltecos, a través del intercambio de saberes; por otro, la labor de la iglesia mediante la pastoral de la tierra, preocupada por las condiciones de los campesinos mayas; y finalmente, las nuevas herramientas metodológicas y tecnológicas. Es en la búsqueda de la soberanía alimentaria (MARTÍNEZTORRES; ROSSET, 2016) donde convergen estos tres elementos. Para lograrla, se ha recurrido a la agroecología como una de las principales herramientas empleadas por la pastoral de la tierra en comunidades de base. Sin embargo, el elemento crucial de la escuela es que no se limita a la producción de alimentos, lo que normalmente ocurre en otras escuelas campesinas donde se provee básicamente de conocimientos técnicos-productivos.

Desde su inicio en la etapa de internado uno de los ejes de formación fue el humanosocial, que incluía la espiritualidad, es decir, la escuela se preocupó no solo por el hacer si no por el ser. Esto permitió establecer un modelo alternativo que no partiera de una lógica productiva; en cambio, la escuela buscó que los participantes volvieran a reconectarse con su entorno y cuestionaran su realidad, a partir de la reconfiguración de su identidad a partir del rescate de su historia. Podemos afirmar, entonces, que la escuela integra una diversidad de propuestas y caminos que, desde un aprendizaje significativo, en su cotidianidad y su planteamiento de vida proponen llegar a la construcción de comunitaria.

En la escuela se llevan a cabo actividades relacionadas con tres aspectos: Espiritual (E); Identitario (I) y productivo (P). Si bien ciertas actividades se desarrollan en una sola dimensión, sea espiritual, identitario o productivo, la escuela busca la interrelación entre las dimensiones (Fig.3). Estos procesos de actividades productivo-espiritual (PE), espiritualidentitario (EI), identitario- productivo (IP) y espiritual- identitario y productivo (EIP), enriquecen la propuesta de la escuela.

\footnotetext{
2 Toda la información en este apartado que no lleva cita bibliográfica, es producto del periodo de trabajo etnográfico, observación participativa y entrevistas realizadas durante la investigación en 2017.
} 


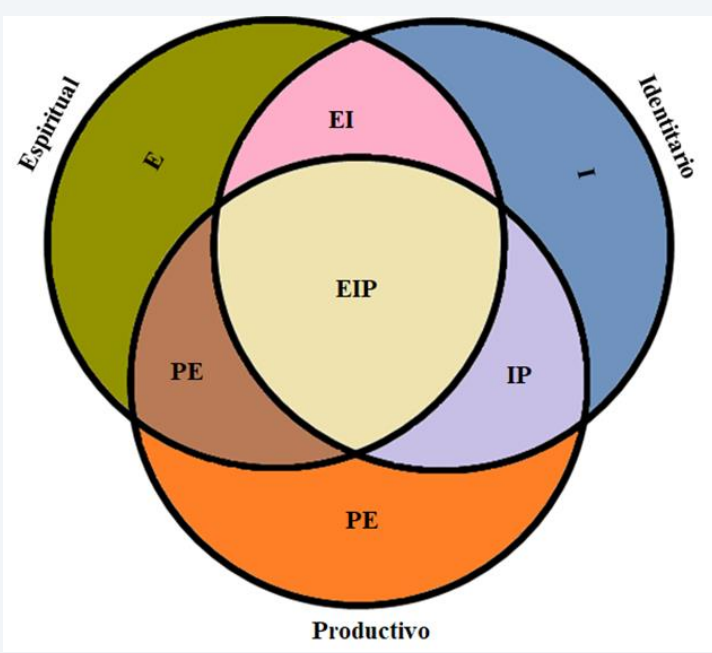

\begin{tabular}{|c|l}
\hline $\mathbf{E}$ & Espiritual \\
\hline $\mathbf{I}$ & Identitario \\
\hline $\mathbf{P}$ & Productivo \\
\hline $\mathbf{E I}$ & Espiritual e identitario \\
\hline $\mathbf{I P}$ & Identitario y productivo \\
\hline $\mathbf{P E}$ & Productivo y espiritual \\
\hline EIP & Espiritual, identitario y productivo \\
\hline
\end{tabular}

Figura 3. Interrelación de dimensiones de las actividades de la escuela. Fuente: elaboración propia.

Ahora bien, comprendiendo la intencionalidad de la escuela a través de estas tres dimensiones, encontramos que la distribución de las actividades es estratégica para interrelacionar dichas dimensiones. En la Figura 4, se ubican los múltiples proyectos y actividades de la escuela en función de su relevancia en el campo de la espiritualidad, la identidad comunitaria y los aspectos productivos. Una actividad puede iniciar siendo principalmente productiva, pero posteriormente desarrollar una estrecha relación con la espiritualidad o con la identidad comunitaria, y viceversa.

\begin{tabular}{|c|l}
\hline EIP & $\begin{array}{l}\text { Baluartes Slow food } \\
\text { Comerciando como hermano }\end{array}$ \\
\cline { 1 - 2 } $\mathbf{P}$ & $\begin{array}{l}\text { Convenio INAH } \\
\text { Día de la Xunán Kab } \\
\text { Día del campesino/a }\end{array}$ \\
\cline { 1 - 2 } $\mathbf{E I}$ & $\begin{array}{l}\text { Fiesta de las semillas } \\
\text { EI }\end{array}$ \\
\cline { 1 - 2 } $\mathbf{E I}$ & $\begin{array}{l}\text { Grupo de epigrafistas } \\
\text { Giras de intercambio }\end{array}$ \\
\cline { 1 - 2 } $\mathbf{P}$ & $\begin{array}{l}\text { Granjas integrales } \\
\text { Medicina tradicional }\end{array}$ \\
\cline { 1 - 2 } $\mathbf{E I P}$ &
\end{tabular}

\begin{tabular}{|c|c|}
\hline EIP & Meliponicultura \\
\hline IP & Mujeres bordadoras \\
\hline IP & Naranjeros de Dzan \\
\hline $\mathbf{P}$ & Prácticas agroecológicas \\
\hline IP & Proyecto vida sana y solidaria \\
\hline IP & Recuperación cerdo criollo \\
\hline IP & Recuperación de especies meliferas \\
\hline EIP & Recuperación de rituales mayas \\
\hline PE & Santuarios ecológicos \\
\hline EI & Teología indígena máyense \\
\hline
\end{tabular}

Figura 4. Clasificación de las actividades de la escuela. Fuente: elaboración propia.

Es importante resaltar que algunas actividades se pueden localizar simultáneamente en los tres ejes, como la meliponicultura.

Con base a lo anterior, la figura 5, ilustra la actividad de la meliponicultura. Está puede ser considerada como una actividad netamente productiva, sin embargo, por medio de la escuela esta actividad adquiere una relación entre las tres dimensiones: productivo por la miel y el 
análisis de la misma, y espiritual e identitaria por el enfoque histórico ritual que hace eco con su cotidianidad a través de la fiesta y celebración de día de la Xunán Kab y la recuperación de aspectos de la medicina tradicional.
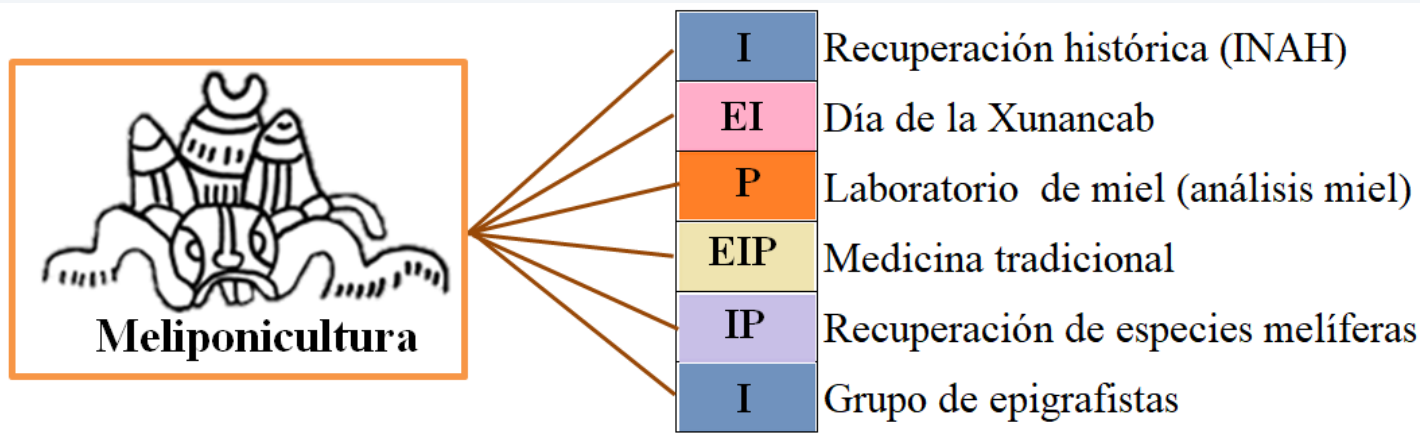

Figura 5. Interrelación de dimensiones en la Meliponicultura. Fuente: elaboración propia.

En resumen, la estrategia de la escuela es generar actividades que correlacionen lo espiritual y la identidad comunitaria con los aspectos productivos. Esto fortalece su convicción en cuanto a la armonía entre estas partes como forma de construcción de un estilo de vida digna y plena, en radical oposición con el enfoque productivista.

\section{b) Construcción de la identidad colectiva}

La identidad colectiva refleja una cierta cohesión de un grupo que asume características que lo identifican frente o en relación con otros grupos o colectivos. Como indica Cabrera (2004), está articulada por un grupo de creencias de una visión sobre "nosotros" - ya sea una visión de un grupo sobre sí mismo o que sujetos externos al grupo tienen sobre él y sus miembros-; y son los significados, la matriz en la que se sostienen esas significaciones sociales a partir de las cuales se construyen colectivamente espacios simbólicos y significaciones.

Sin embargo, habría que agregar que además de la construcción de subjetividades simbólicas se debe rescatar la importancia de las prácticas sociales en la construcción de identidades colectivas (TEJERINA, 1999). Los procesos por los que se construye una identidad colectiva pasan por un mecanismo de reconocimiento inicialmente interno y posteriormente en interacción con su contexto, lo que permite reconocer lo que se tiene en común y facilita la decisión de actuar colectivamente. (RODRÍGUEZ-ARECHAVALETA, 2010).

En la escuela $U$ Yits $K a$ 'an la propuesta de la construcción de identidad colectiva está basada en lo comunitario, se asienta no sólo en la oposición o resistencia a otros grupos, 
métodos o sistemas de producción, sino también en prácticas y saberes latentes y manifiestos de los campesinos, campesinas e indígenas, la recuperación de identidades colectivas vinculadas a lo campesino-indígena y a lo originario. En el caso específico de la escuela, la construcción de identidad colectiva transita alrededor de ser campesino/a-indígena-mayayucateco, y se expresa en la cotidianidad del trabajo.

Ser maya es ser campesino, ser maya es trabajar la tierra, por ejemplo, costurar, atender los animales [...] pues nosotros de ahí nacimos, ahí crecimos y pues también ya están grandes mis hijos, y están haciendo lo mismo (TORRES, M., comunicación personal, 29 de marzo de 2017).

La identidad se genera a partir de intereses individuales, pero también de la dignificación del ser maya, de valorar el pasado y entenderse ellos mismos como resultado de toda esa herencia. La dignificación a partir de la historia es importante porque se hace un reconocimiento de lo que era antes ser maya, pero se ubican también en la era moderna con todas sus contradicciones.

Podemos hablar, no sé, a lo mejor de dos momentos de los mayas, la historia que traemos atrás que es una historia de grandeza y yo creo que de ahí nos debemos de basar para no sentir vergüenza, pena de ser indígena $[\ldots]$ entonces si nos remontamos un poquito más atrás los mayas han sido una civilización muy grande [...] En algunos momentos la gente piensa "¡ay! Los mayas, las grandes pirámides, Chichen, Uxmal y otras". Pero no, los mayas todavía seguimos, los mayas estamos aquí. Entonces para mí, el ser maya es símbolo de grandeza, es símbolo de sabiduría, de conocimientos, de muchas cosas que tienen que ver con la vida misma (DZIB, A., comunicación personal, 29 de junio de 2017).

El ser maya ha pasado de ser motivo de vergüenza a orgullo, asimilación de la historia y empoderamiento personal y colectivo. Además, esta percepción de la identidad maya tiene una gran implicación espiritual.

\section{c) Espiritualidad}

Hablar de la espiritualidad es un tema complejo y abstracto, sin embargo, en este momento en que la humanidad atraviesa por una crisis civilizatoria, la espiritualidad se erige como opción frente al mundo materialista en el que vivimos, como fuente para la construcción de esperanza. Una de las fuentes de espiritualidad se encuentra en las cosmovisiones y prácticas de los pueblos originarios (TOLEDO, 2003). 
La espiritualidad es algo muy importante para mí [...] es algo que no se ve, pero si tienes esa fe lo puedes sentir, yo así lo siento me concentro mucho y cierro mis ojos para sentir y sí se siente, no se ve, pero se siente, pero aparte de eso la espiritualidad acá entre los mayas es muy conocida, por ejemplo, con la primicia del Saká que hacemos nosotros en nuestras milpas, en nuestras parcelas (CASTILLO, N., comunicación personal, 22 de julio de 2017).

Podemos ver que existe una concepción particular de la espiritualidad, que no se limita al espíritu o la piedad cristiana, sino que se traduce en acciones concretas y en una relación de respeto y vida con la naturaleza, misma que va más allá de lo humano.

\begin{abstract}
¿Dónde está la religión? [...] Un apicultor esperando que llueva y llegue la floración, lo veo cargando su mochila y comprando cajas para las abejas. Ese es el tipo de espiritualidad que se necesita, no leer y leer los versículos de la biblia. Porque en lo espiritual si no llueve no te pongas a hacer rogativas, porque de por sí no va a llover; mejor organízate y ve cómo hacer un pozo o un sistema de riego. Eso es el tipo de espiritualidad que se necesita y no rezar tantas Aves Marías (XIU, B., comunicación personal, 7 de Julio de 2017).
\end{abstract}

Como señala Toledo (2003):

Los pueblos indios mantienen una visión del mundo de la que la percepción racionalista y utilitaria que prevalece en los espacios industriales ya no dispone. Para las culturas indígenas la naturaleza no sólo es una respetable fuente productiva, es el centro del universo, el núcleo de la cultura y el origen de la identidad étnica. Y en la esencia de este profundo lazo prevalece la percepción de que todas las cosas, vivas y no vivas, están intrínsecamente ligadas con lo humano (p.65).

La espiritualidad se entiende entonces como una conexión profunda, una crítica $\mathrm{Al}$ comportamiento del ser humano y a ciertas conductas antropocéntricas.

Es importante la espiritualidad porque el ser humano tiene partes: el cuerpo, mente y espíritu y hay que estar juntos; y es unirlo para que tenga forma y para que las cosas funcionen. La vida es un espíritu; la vida de las plantas y la vida de los seres humanos son un espíritu y es algo que nos persigue en cada momento y no puede dejar de existir (XIU, B., comunicación personal, 7 de Julio de 2017).

\title{
d) Madre Tierra
}

Un concepto que ejemplifica la integración de la espiritualidad maya y su identidad es la construcción subjetiva simbólica de que la tierra es su Madre. Una representación simultánea de la capacidad generadora de vida, proveedora de alimentos y cobijo. 
En relación a lo anterior tenemos que comprender que para los mayas la esfera terrestre tiene vida. La tierra tiene la capacidad de sostener la vida, tanto la nuestra como de todo lo que existe; pero no es un receptáculo como se entiende por la visión antropocéntrica occidental. La perspectiva de grupos indígenas como los Aymara en Bolivia, refieren que la Tierra es un ser vivo y por ello puede reproducir la vida. Se identifica con el termino de Madre tierra debido al hecho de que los seres humanos surgen de la vida de la tierra, particularmente de la matriz de vida que la tierra tiene. Por eso y en una forma de interpretación simbólica, la Madre Tierra tiene que defenderse, cuidarse y respetarse (COMINS, 2016).

Recuerdo desde niño que mi abuelo siempre decía que se tiene que dar de comer a la tierra a través de las ofrendas, yo no entendía por qué lo decía, pero con el tiempo y ahora que él ya murió, veo que aprendí mucho de él, la Madre Tierra para mí es como mi madre que me dio la vida, pero la tierra es como todas las madres, no todas las mamás son queridas por sus hijos $[\ldots]$ a pesar que de ella surge la vida ella sufre todo, todo viene del seno del útero, para mí muy semejante a la tierra, soy parte del fruto del útero de la tierra (XIU, B., comunicación personal, 7 de Julio de 2017).

La vida no debe entenderse en el sentido occidental de que cada sujeto tiene vida propia, sino de la vida como un complejo entramado de relaciones. Es una visión de vida comunitaria, colectiva, identitaria, que nuestra Madre Tierra nos comparte.

Por lo tanto, se debe asumir que somos nosotros los que pertenecemos a nuestra Madre Tierra, que es nuestra cuna, nos alimenta y nos guarda al fallecer, cuando hemos de reintegramos a ella para continuar vivos de alguna otra forma. Se rompe así con la idea occidental de que somos nosotros quienes tenemos la tierra como una posesión. Nosotros somos de la Madre Tierra y ella no es nuestra.

La Madre Tierra, bueno yo creo que es uno de los elementos tan primordiales que tenemos aquí [...] es muy importante que lo cuidemos, que tengamos presente de que nuestra Madre Tierra vive, y que hay que cuidarla, hay que respetarla todos los días para que nos pueda seguir dando el sustento cada día. Y que al final de cuentas ahí vamos también a descansar. Tal vez ahí formemos parte de los abonos que necesita la propia tierra para seguir regenerándose ¿no? Entonces la Madre Tierra es símbolo de respeto y de vida para las poblaciones indígenas, como digo no hablo solamente de los mayas sino de todas las poblaciones indígenas y no solo de México sino del mundo, porque hay muchos pueblos indígenas que siguen respetando el sol, la tierra, el agua, el aire porque son elementos muy importantes, vitales para nuestra existencia (DZIB, A., comunicación personal, 29 de junio de 2017). 
e) Ma'alob Kuxtal o "Buen vivir"

Según el pueblo Maya Tseltal, el Lekil Kuxlejal (la vida buena), es una filosofía de vida. Se refiere a una relación de armonía entre la humanidad y la naturaleza; se relaciona con el mejoramiento de la vida, sin limitarse a los bienes materiales, si no que abarca valores humanos, morales y espirituales y sobre todo remarca la importancia de lo colectivo ante lo individual. (HOUTART, 2014). La visión maya yucateca de Ma'alob Kuxtal o "buen vivir," de la vida buena, plena y digna, consiste en una serie de principios filosóficos de vida, que tiene un significado como símbolo de grandeza, orgullo, sabiduría, honor. Es evidencia de una cultura viva a través de su lengua y al interior de su territorio.

El buen vivir no solo se preocupa por el tener sino por el ser. Por lo tanto, podemos decir que es un concepto filosófico complejo que ha sido construido históricamente, no es lineal y, en consecuencia, está en constante resignificación. El buen vivir tiene que ver entonces con satisfacer necesidades, lograr una calidad de vida, tener una muerte digna, pero también tiene que ver con el amor, la paz y la armonía con la naturaleza, para el bien de la humanidad (BRETÓN SOLO DE ZALDÍVAR, 2013). Como señala Hidalgo (2011), el buen vivir es un reflejo de lo que debería ser la misión de la humanidad para lograr una vida armónica.

Josef Esterman (citado en GIRALDO, 2014), afirma que en el buen vivir se articulan los principios de relacionalidad, que se refiere a que todo está conectado e interrelacionado con lo demás; la complementariedad, que nos dice que nada existe por sí mismo; la correspondencia, donde todos los aspectos que componen la realidad se correlacionan en ambas direcciones, es decir, es recíproco.

La visión del buen vivir implica dimensiones culturales, ambientales, sociales y económicos, es una propuesta a rehacer la vida a partir de la construcción de alternativas que implican el derecho a la libre determinación de los pueblos indígenas, desarrollando sus modos de vida, orientados al bienestar colectivo (ZAMORA, 2016). Este buen vivir no puede equipararse a la calidad de vida a la que apela la lógica capitalista y desarrollista, una lógica de exceso y derroche:

Ha cambiado mucho mi casa, pero no cosas así para ser "ricos", porque sabes que la vida no es la riqueza total, si no aprender a vivirla bien, porque pues yo siempre sigo también como de antes, soy una persona sencilla, pero pues valoro muchas veces los cambios, que ha tenido mi vida últimamente, he visto que hay cambios muy significativos, a nivel personal, no tanto económicamente como te digo, pero a nivel personal, porque pues me he sensibilizado más con la naturaleza, no como antes, 
hoy me doy cuenta de que hay que estar bien con la naturaleza, no es sentirse bien con uno mismo, sino que con todo lo que te rodea, los animales, los árboles porque son parte de nuestro mundo (CASTILLO, N., comunicación personal, 22 de julio de 2017).

Según Giraldo (2014):

A lo que aspira el Buen Vivir es a construir una ontología relacional, por la cual nos aprehendamos a nosotros mismos, como miembros de una gran comunidad ligada en redes de interdependencia mutua y que actuemos en consecuencia. Que reconozcamos que vivimos en un mundo de simbiosis, en donde cada quien es incompleto y requiere de "lo otro" para complementarse (p.113).

f) El despertar de la conciencia

Podemos decir que hay un antes y un después de participar en la escuela; al preguntarle a las personas cuáles fueron los cambios más significativos, no solo se refieren a los activos productivos sino a la forma en que cuestionan su realidad, cuestionan las formas de producir, pero también la forma de tomar decisiones.

La escuelita me ha dado la satisfacción de ver el mundo de otra forma, porque no solo es el pueblo, sino que involucra todo [...] como quien dice ver bien y analizar bien [...] Entonces me enseñó a ser más precavido en todo. Me enseñó a abrir los ojos y la mente, estoy muy agradecido porque me enseñó a defender lo que es mío, nosotros defendemos lo que es de nosotros: nuestras herencias, nuestras costumbres (CASTILLO, N., comunicación personal, 22 de julio de 2017)

Este cuestionamiento permite que los sujetos se posicionen respecto a los demás y de su papel en la vida social de su comunidad de manera fraterna y solidaria.

La escuela nos ha dado un gran beneficio, saber cosas que son de nosotros, que debemos de defender nosotros los mayas, hay muchas cosas que nos pertenecen, que nos están quitando. [...] También hemos aprendido que hay que ser generoso con la gente, hay que enseñar a la gente, hay que platicar con la gente no hay que darles las espaldas a la gente que lo necesite $[\ldots]$ a veces son personas de otra religión, pero allá en la escuela todos somos hermanos. [...] Si tú tienes un poco tienes que compartir, tienes que dárselo a los pobres. [...] tenemos que darles un poco, a nuestros semejantes (TORRES, M., comunicación personal, 29 de marzo de 2017).

La reconstrucción y permanencia de la comunidad está relacionada a la enseñanza que se les da a los hijos, es decir, involucra a distintas generaciones, quienes deben tomar un papel activo en esta transformación. En la escuela hacen un llamado urgente a enseñar a los hijos e 
hijas a amar la tierra, no sólo a través de la educación oficial del Estado sino a través de un verdadero acercamiento vivencial a ella.

Ver el trabajo del campo no como un castigo, si no como una forma muy digna de buscarse la vida y de llevar a la mesa los alimentos. Entonces es importante inculcarles esto a los niños, a los nietos llevarlos desde muy pequeñitos al campo, que se aprendan a revolcar en la tierra, ensuciarse las manos y a convivir con el campo (DZIB, A., comunicación personal, 29 de junio de 2017).

Por esta razón es muy importante la permanencia de los procesos que se detonaron en la escuela, lo que necesariamente tiene que incluir a las generaciones que están por venir, en medio de las contradicciones con el mundo moderno, en medio de la incertidumbre que deriva de las enseñanzas de la escuela oficial. Sin embargo, frente a lo que el maestro enseña, tendremos que ver qué dice un alumno.

En realidad, no sabemos qué pasara, pero por ahora tenemos una buena generación de jóvenes [...] Por más buenas que sean las cosas todas las cosas materiales se acaban, y las cosas que son como los ideales duran un poco más; lo cultural, lo espiritual permanecen, siempre ha convertido la historia de la gente, es la historia que no se va a olvidar nunca (XIU, B., comunicación personal, 7 de Julio de 2017).

\section{Conclusiones}

Uno de los aspectos más relevantes del carácter espiritual de la visión maya radica en su proceso de liberación interna, este despertar de la conciencia que se genera desde lo personal pero que incluye un concepto de comunión con los demás, así como con la Madre Tierra.

Este marco subjetivo permite mirar las cosas de otra forma y posicionarse al respecto. Por ello consiste no únicamente en modificar su hacer cotidiano sino su ser interior. Proceso que al suceder en varios sujetos construye un ambiente de intenso intercambio colectivo. Se crea una colectividad a partir de esto, en resumen, el trabajo de espiritualidad en la identidad conduce a una liberación del pensamiento. Esta construcción ahora colectiva genera un sentido de esperanza en el futuro.

Esta es la explicación de que se hayan encontrado tantos testimonios profundos, honestos y a la vez tan cotidianos, prácticos y aplicados.

La vida buena, plena y digna, que identifican como paradigma los mayas, está constituida de varios elementos interrelacionados de forma compleja pero cuyas claves se pueden deducir a partir del trabajo de la escuela. Tiene como objetivo general construir un 
ambiente donde todos los sujetos que de alguna forma participan en la escuela pueden vivir "la vida buena, plena y digna" o Ma'alob Kuxtal. Identificamos los siguientes aspectos: El primero, integrar en los proyectos además de los conocimientos productivos, las dimensiones espirituales e identitarias, esto es, aprender a "Sentipensar" lo que sucede y tomar acciones al respecto. El segundo, la priorización de lo colectivo sobre lo individual para la solución de los problemas. A partir del desarrollo de estrategias de trabajo que busquen la emancipación y la autoconstrucción en vez de la toma de propuestas que se plantean como alternativas al sistema pero que crean otras dependencias; y en tercero, reconstruir el "ser" para que, a partir de ese proceso individual, se modifique el hacer de las actividades. Esto permite un nivel de adaptación no solamente tecnológico sino también conceptual y estructural. En otros términos, se trata de analizar desde el ser subjetivo de cada participante para hacer las cosas según su particular circunstancia y generar una construcción humana, diversa y etnocentrada.

Podemos decir que de manera natural los sujetos conforme trabajan su espiritualidad y su reconstrucción identitaria, van buscando proyectos gradualmente de mayor complejidad pero que les hacen más sentido. Es decir, la oferta de los proyectos en la escuela obedece tanto a la necesidad y visión identitaria como al deseo de vivir de una manera más cercana la "vida buena, plena y digna" o Ma'alob Kuxtal.

Este proceso de diversificación de las actividades productivas no se basa solamente en hacer sustitución de insumos, cambiar de modelo de producción o replicar prácticas agroecológicas. La escuela campesina de Agricultura Ecológica U Yits Káan revalora y reinventa la agroecología desde su identidad étnica. Para modificar su visión de la vida y aplicarlo en su cotidianidad, en forma colectiva.

\section{REFERENCIAS}

Álvarez, F. (2010). Escuelas campesinas de agroecología: una estrategia de desarrollo endógeno sustentable en el municipio de Tuluá. Revista de Investigación Agraria y Ambiental, 1(2), 51-63. DOI: https://doi.org/10.22490/21456453.898

Arias, M. M., y Carrasco, B. (1998). La palabra de Dios se hizo indio: teología y práctica de una catequesis inculturada y liberadora. Una propuesta desde Oaxaca, México. México: Ediciones DABAR.

Berryman, P. (2003). Teología de la Liberación. México: Siglo veintiuno editores.

Boero, M. (1984). La Iglesia de América Latina y la Teología de la Liberación. Revista Araucaria de Chile, (28), 31-35. Recuperado de https://www.archivochile.com /Cultura_Arte_Educacion /araucaria/ araucaria28.pdf

Boff, L. (1990). Nueva evangelización. Argentina: Editorial LUMEN. 
Bretón Solo de Zaldívar, V. (2013). Etnicidad, desarrollo y "Buen Vivir": Reflexiones críticas en perspectiva histórica. Revista Europea de Estudios Latinoamericanos y del Caribe, (95), 71-95. DOI: https://doi.org/10.18352/erlacs.9231

Cabrera, D. (2004). "Imaginario social, comunicación e identidad colectiva". En el dialogo: "Comunicación y diversidad cultural", Forum Barcelona. Universidad de Navarra. Barcelona, España. Recuperado de http://www.portalcomunicacion.com/dialeg/paper/pdf/143_cabrera.pdf

Campos, C. G. (2007). Teología máyense. El discurso sistémico de lo sagrado por la defensa de derechos indígenas (Tesis de maestría). Universidad de Ciencias y Artes de Chiapas. Chiapas, México.

Castillo, N. (22 de julio de 2017). Entrevista promotor Nicolás, Maní. [Archivo de audio]. Copia en posesión del autor. México

Castro Guzmán, M., Méndez Cano, J., y Centurión, K. (2017). Maní, un pueblo originario de Yucatán y el Acto de Fe en el siglo XVI: Un análisis retrospectivo entre lo sacro y pagano. Revista Salud y Bienestar Social, 1(1), 115-127. Recuperado de http://www.revista.enfermeria.uady.mx/ojs/index.php/Salud/ article/ view/13

Ceballos, L. A. (2014). Experiencias y avances actuales: Escuela de agricultura ecológica U Yits Ka'an. En Mata. G. B (Coord.), Escuelas campesinas en México. Diagnósticos y aportes a la educación rural alternativa (pp. 49 - 54). Estado de México: Universidad Autónoma Chapingo

Comins, M. I. (2016). La filosofía del cuidado de la tierra como Ecosofía. Daimon. Revista Internacional de Filosofía, (67), 133-148. DOI: https://doi.org/10.6018/201501

Dzib, A. (29 de junio de 2017). Entrevista, locutor Xepet, Escuela. [Archivo de audio]. Copia en posesión del autor. México

Estermann, J. (2012). Crisis civilizatoria y Vivir Bien. Polis, Revista Latinoamericana, (33), 122. Recuperado en https://journals.openedition.org/polis/8476

Galeano, E. (1991). Memoria del fuego I. Los nacimientos. México: Siglo XXI editores.

Giraldo, O. F. (2014). Utopías en la era de la supervivencia. Una interpretación del Buen Vivir. México: Editorial ITACA.

Hernández, G. F. (2015). Sistematización de la experiencia de la Escuela de Agricultura Ecológica U Yits Ka'an y su efecto sobre los medios de vida de las familias participantes en el municipio de Maní, Yucatán, México (Tesis de maestría). Centro Agronómico Tropical de Investigación y Enseñanza, Turrialba, Costa Rica. Recuperado de http://repositorio.bibliotecaorton.catie.ac.cr/handle/11554/8214

Hidalgo, F. F. (2011). Buen vivir, Sumak Kawsay: Aporte contrahegemónico del proceso andino. Utopía y Praxis Latinoamericana, 16(53), 85-94. Recuperado de https://www.redalyc.org/articulo.oa?id $=27919220008$

Holt-Giménez, E. (2008). Campesino a campesino. Voces de Latinoamérica. Movimiento campesino a campesino para la agricultura sustentable. Managua, Nicaragua: SIMAS

Houtart, F. (2014). El concepto de Sumak Kawsay (Buen vivir) y su correspondencia con el bien común de la humanidad. En Delgado, G.C.(Coord.), Buena vida, buen vivir: imaginarios alternativos para el bien común de la humanidad. (pp. 97-123). México: Universidad Nacional Autónoma de México, Centro de Investigaciones Interdisciplinarias en Ciencias y Humanidades. Recuperado de http://biblioteca.clacso.edu.ar/Mexico/ceiich-unam/20170503034423/pdf_1508.pdf

Kú-May, S. (2005). Los Xiu: Una compilación descriptiva en su encuentro con los españoles (Tesis de Licenciatura). Universidad Autónoma Metropolitana, México. Recuperado de http://148.206.53.84/tesiuami /UAMI12143.pdf

López, B. E. (2013). Teología de la creación desde el mundo indígena: "El sueño de Dios en la creación humana y del mundo". Revista Iberoamericana de Teología., IX (17), 87- 
102. Recuperado de

http://revistas.ibero.mx/ribet/uploads/volumenes/18/pdf/eologia_17_Final_para_web.pdf $\underline{\mathrm{h}}$

Macossay, V. M., Aguilar, C. W., Castillo, C. J., Ceballos, L. A., y Zapata, C. R. (2005). La Escuela de Agricultura Ecológica U Yits Ka’an de Maní, Yucatán. Diez años de trabajo. Revista de Geografía Agrícola, (35), 91-104. Recuperado de https://www.redalyc.org/pdf/757/75703505.pdf

Martínez-Torres, M. E., y Rosset, P. M. (2016). Diálogo de saberes en La Vía Campesina: Soberanía alimentaria y agroecología. Espacio Regional, Revista de estudios sociales 1(13), 23-36. Recuperado

https://www.researchgate.net/publication/308145837_DIALOGO_

DE_SABERES_EN_LA_VIA_CAMPESINA_SOBERANIA_ALIMENTARIA_Y_AGROECOLOGIA

Mata, G. B. (2013). Nuestro caminar con las escuelas campesinas. En Mata, G. B. (Coord.), Escuelas campesinas: 10 años en movimiento (pp. 23-44). Estado de México: Universidad Autónoma Chapingo

Melecio, M. A. (2015). Modificaciones en sistemas educativos en la educación popular: perspectivas de los maestros de la Escuela de Agricultura Ecológica U Yits Ka'an en Yucatán, México (Tesis doctoral). Universidad de Granada, Granada, España. Recuperado de https://digibug.ugr.es/handle/10481/43392

Muñoz, S. P. (2013). Educación, desarrollo y saberes campesinos en Atenco, México. En Mata, G. B. (Coord.), Escuelas campesinas: 10 años en movimiento (pp. 213-224). Estado de México: Universidad Autónoma Chapingo

Rodríguez-Arechavaleta, C. M. (2010). De la estructura de oportunidades políticas a la identidad colectiva. Apuntes teóricos sobre el poder, la acción colectiva y los movimientos sociales. Revista, Espacios Públicos, 13(27), 187-215. Recuperado de https://www.redalyc.org/pdf/676/67613199012.pdf

Segundo, G. (1978). Teología de la Liberación: intento de síntesis. Lumen Vitae, 33, 205-228. Recuperado http://www.seleccionesdeteologia.net/selecciones/llib/vol19/76/076_galilea.pdf

Suárez, C. M. de G. (2014). El convento de Maní, Yucatán, en 1588. Boletín de Monumentos Históricos, Tercera época (31), 78-92. Recuperado de http://boletincnmh.inah.gob.mx/boletin/boletines/bmh\%2031-5.pdf

Tejerina, B. (1999). El poder de los símbolos: Identidad colectiva y movimiento etnolingüístico en el País Vasco. REIS, Revista Española de Investigaciones Sociológicas, (88), 75-105. DOI: $10.2307 / 40184204$

Toledo, V. M. (2003). Hacia una sociedad sustentable. En Toledo, V. M. (2003), Ecología, Espiritualidad y Conocimiento, de la sociedad del riesgo a la sociedad sustentable. (66130). México: Programa de las Naciones Unidas para el Medio Ambiente. Recuperado de http://atlas.umss.edu.bo:8080/jspui/handle/123456789/544

Torres, M. (29 de marzo de 2017). Entrevista promotora, localidad Maní. [Archivo de audio]. Copia en posesión del autor. México

Xiu, B. (7 de julio de 2017). Promotor escuela Xiu. [Archivo de audio]. Copia en posesión del autor. México

Zamora, L. C. B. (2016). Sa'el jun ontonal, la búsqueda del bienestar, la autonomía y la paz desde Las Abejas de Acteal. Polis, Revista Latinoamericana, (43), 1-17. Recuperado en https://journals .openedition.org/polis/11632 


\section{SOBRE OS AUTORES:}

\section{Rosa López Valentín}

M.Sc. (ECOSUR). El Colegio de la Frontera Sur (ECOSUR), México, Programa de Maestría en Ciencias en Recursos Naturales y Desarrollo Rural, y Grupo de Investigación sobre la Masificación de la Agroecología. Becaria de CONACYT. E-mail: rosalovale@ gmail.com

(iD http://orcid.org/0000-0003-0691-245X

\section{Peter Michael Rosset}

Ph.D. (University of Michigan, EEUU). El Colegio de la Frontera Sur (ECOSUR), México, Departamento de Agricultura, Sociedad y Ambiente, Grupo Académico de Agroecología, y Grupo de Investigación sobre la Masificación de la Agroecología. Universidad Estatal de Ceará (UECE), Brasil, Programa de Pós-Graduação em Sociologia (PPGS). Becario de BPVFUNCAP. E-mail: prosset@ecosur.mx

(iD http://orcid.org/0000-0002-1253-1066

\section{Carla Beatriz Zamora Lomelí}

Ph.D. (COLMEX). El Colegio de la Frontera Sur (ECOSUR), México, Departamento de Agricultura, Sociedad y Ambiente, Grupo Académico de Estudios Socioambientales y Gestión Territorial. E-mail: czamora@ecosur.mx

iD http://orcid.org/0000-0003-4089-2659

\section{Omar Felipe Giraldo Palacio}

Ph.D. (CHAPINGO). El Colegio de la Frontera Sur (ECOSUR), México, Departamento de Agricultura, Sociedad y Ambiente, Grupo Académico de Agroecología, y Grupo de Investigación sobre la Masificación de la Agroecología. Becario Cátedra de CONACYT. Email: omarfgiraldo@gmail.com

iD http://orcid.org/0000-0002-3485-5694

\section{María Virginia González Santiago}

Ph.D. (ENAH). Universidad Autónoma de Chapingo (CHAPINGO), México, Programa de Agroecología. E-mail: marvirginia2000@yahoo.com.mx

iD http://orcid.org/0000-0002-9726-7136

Recebido em: 07 de novembro de 2019

Aprovado em: 29 de janeiro de 2020

Publicado em: 01 de abril de 2020 